\title{
Nonuniform ground state for the Bose gas
}

\author{
András Sütő \\ Research Institute for Solid State Physics \\ Hungarian Academy of Sciences \\ P. O. Box 49, H-1525 Budapest 114 \\ Hungary \\ E-mail: suto@szfki.hu
}

\begin{abstract}
We study the ground state $\Psi_{0}=\sum a_{X}|X\rangle$ of $N$ hard-core bosons on a finite lattice in configuration space, $X=\left\{x_{1}, \ldots, x_{N}\right\}$. All $a_{X}$ being positive, the ratios $a_{X} / \sum a_{Y}$ can be interpreted as probabilities $P_{a}(X)$. Let $E_{0}$ denote the energy of the ground state and $|\partial X|$ the number of nearest-neighbour particle-hole pairs in the configuration $X$. We prove the concentration of $P_{a}$ onto $X$ 's with $|\partial X|$ in a $\sqrt{\left|E_{0}\right|}$ neighbourhood of $\left|E_{0}\right|$, show that the average of $a_{X}$ over configurations with $|\partial X|=n$ increases exponentially with $n$, discuss fluctuations about this average, derive upper and lower bounds on $E_{0}$ and give an argument for off-diagonal long-range order in the ground state.
\end{abstract}

PACS: 05.30.Jp, 05.50.+q, 75.10.Jm 


\section{Introduction}

Bosons on a lattice interacting via an infinite on-site repulsion (hard-core bosons) represent a system of double interest. This is the simplest example of an interacting Bose gas and, thus, the most promising candidate for a rigorous treatment of Bose-Einstein condensation of interacting particles. The model is also known to be equivalent to a system of $\frac{1}{2}$ spins (Matsubara and Matsuda 1956) coupled via the $X$ and $Y$ and possibly the $Z$ components of neighbouring spins and exposed to an external magnetic field in the $Z$ direction. Ordering of the planar component of the spins is equivalent to Bose-Einstein condensation or the appearance of an off-diagonal long-range order (ODLRO, Yang 1962) in the system of bosons. Apart from some exceptions, like bounds on the density of the condensate (Tóth 1991) or the discussion of the model on complete graphs (Tóth 1990, Penrose 1991), the most interesting rigorous results were formulated in spin terminology and obtained by the use of a particular symmetry, reflexion positivity (Dyson et al 1978, Kennedy et al 1988, Kubo and Kishi 1988). This introduced limitations on the value of the external field (zero field) and the lattice type (essentially hypercubic lattices). Expressed in terms of a boson gas, ODLRO was shown only at half filling on hypercubic lattices: in the ground state in dimensions $\geq 2$, and for positive temperatures above two dimensions. The proof of ordering does not offer much insight into the structure of the state. The only case in which details are known is the mathematically nice but physically trivial example of the complete graph (Tóth 1990, Penrose 1991).

In this paper we provide informations about the ground state $\Psi_{0}$, valid for very different lattices and arbitrary particle densities. The main result is the proof of a strong nonuniformity of $\Psi_{0}$ in configuration space. It appears mathematically in the form of a large deviation principle and makes the ground state resemble a thermal Gibbs distribution of a classical gas on the same lattice. It also leads to an approximate expression for $\Psi_{0}$ and to an argument for Bose-Einstein condensation in the ground state in two and higher dimensions.

Let $\mathbf{L}$ be an infinite lattice which, for the sake of simplicity, will be supposed to be regular with a constant coordination number (degree) $k$. Throughout the paper $\Lambda$ denotes a finite connected part of $\mathbf{L}$ taken with periodic boundary conditions so as to keep the degree constant (not really 
essential). The Hamiltonian we are going to study in detail is

$$
H_{0}=-\sum_{\langle x y\rangle}\left(b_{x}^{*} b_{y}+b_{y}^{*} b_{x}\right)
$$

We write $x, y, \ldots$ for the vertices (sites) and $\langle x y\rangle$ for the edges (nearestneighbour pairs); $b_{x}^{*}$ and $b_{x}$ create, resp., annihilate a hard-core boson at $x$. Boson operators at different sites commute with each other while

$$
b_{x}^{*} b_{x}+b_{x} b_{x}^{*}=1
$$

accounts for the hard-core condition. Correspondence with spin models is obtained by setting $b_{x}=S_{x}^{-}$and $b_{x}^{*}=S_{x}^{+}$. The Hamiltonian conserves the number of bosons,

$$
N=\sum_{x \in \Lambda} n_{x}=\sum_{x \in \Lambda} b_{x}^{*} b_{x}
$$

and is also invariant under particle-hole transformation. We can, therefore, fix $N$ so that $\rho=N /|\Lambda|$ is between 0 and $\frac{1}{2}$. (Here and below, if $A$ is a finite set, $|A|$ stands for the number of its elements.) Let $X, Y, \ldots$ denote $N$-point subsets of $\Lambda$, called also configurations. A convenient basis is formed by the states

$$
|X\rangle=\left(\prod_{x \in X} b_{x}^{*}\right)|0\rangle
$$

where $|0\rangle$ is the vacuum state. According to the Perron-Frobenius theorem, there is a single ground state $\Psi_{0}$ whose all coefficients in the basis (4) are positive. Let $a=\left(a_{X}\right)$ denote the vector of these coefficients. Since the unique source of energy is hopping constrained by an on-site exclusion, we can expect that $a_{X}$ increases, at least 'in average', with an increasing number of nearest neighbour particle-hole pairs. More precisely, let $\partial X$ denote the set of nearest-neighbour particle-hole pairs for $X$ (the 'boundary' of $X$ ) and $\Omega_{n}$ the ensemble of configurations $X$ with 'boundary length' $|\partial X|=n$. It is easily seen that

$$
|\partial X|=\left\langle X\left|H_{0}^{2}\right| X\right\rangle=\langle X|\Delta| X\rangle
$$

where

$$
\Delta=\sum_{\langle x y\rangle}\left[n_{x}\left(1-n_{y}\right)+n_{y}\left(1-n_{x}\right)\right]
$$

is the diagonal part of $H_{0}^{2}$, and $\left|\Omega_{n}\right|$ is the multiplicity of the eigenvalue $n$ of $\Delta$. We expect that the $X$-dependence of $a_{X}$ appears mainly through $|\partial X|$, 
and the average

$$
\alpha_{n}=\frac{1}{\left|\Omega_{n}\right|} \sum_{X \in \Omega_{n}} a_{X}
$$

is some increasing function of $n$. We shall indeed find that $\alpha_{n}$ rapidly grows with $n$ - exponentially fast for $n$ of the order of $N$ and even faster for smaller $n$. To make a comparison with the free Bose gas, let us notice that the ground state of the latter reads

$$
\Psi_{\text {free }}=|\Lambda|^{-N / 2} \sum_{\left\{m_{x} \geq 0\right\}_{x \in \Lambda}: \sum m_{x}=N} \sqrt{\frac{N !}{\prod_{x \in \Lambda} m_{x} !}} \prod_{x \in \Lambda}\left(a_{x}^{*}\right)^{m_{x}}|0\rangle
$$

where $a_{x}^{*}$ is the ordinary boson creation operator at $x$. If we project out the part with no particle encounters, we find const $\times \sum|X\rangle$, the sum going over the basis (4). This uniform sum is qualitatively very different from what we actually find for the ground state, and the energy of this state will also be found to be extensively higher than that of the ground state.

We start, in Section 2, by studying the probability measure

$$
p_{a}(n)=\frac{\left|\Omega_{n}\right| \alpha_{n}}{\sum_{m}\left|\Omega_{m}\right| \alpha_{m}} .
$$

We show that the mean value according to $p_{a}$ is $\left|E_{0}\right|$, the modulus of the ground state energy, while the mean square deviation $D_{a}^{2}$ is of order $N$, so that $p_{a}(n)$ is concentrated onto integers with $|n-| E_{0}||$ of order $\sqrt{N}$. In Section 3 we evoke a large deviation principle for the Ising model on the same lattice, having the approximate form

$$
q(n) \equiv \frac{\left|\Omega_{n}\right|}{\sum_{m}\left|\Omega_{m}\right|} \propto e^{-\frac{(n-M)^{2}}{2 D^{2}}} .
$$

Here $M=\overline{|\partial X|}$, the (arithmetic) mean of boundary lengths among $N$-point configurations (and also the modulus of the energy of the projected freeboson ground state) and $D^{2}$ is the corresponding mean square deviation of $|\partial X|$. We derive a formula for $D^{2}$ as a function of $\rho$ and $k(M=k \rho(1-\rho)|\Lambda|$ can be obtained trivially). In Section 4 we use the results on $p_{a}$ and $q$ to give an approximate expression for $\alpha_{n}$ in terms of $E_{0}, D_{a}, M$ and $D$. We present an argument for the monotonicity of $\alpha_{n}$ and deduce from it upper and lower bounds on $D_{a} / D$, predicting $\alpha_{n}=$ const if $\left|E_{0}\right| / M=1$ asymptotically (as $|\Lambda|$ and $N$ go to infinity). The variational treatment in Section 5 shows 
that $\left|E_{0}\right| / M>1$ in the thermodynamic limit. A notable exception, with $\left|E_{0}\right|=M=N(|\Lambda|-N)$, is the complete graph where by permutational symmetry we actually have $a_{X}=$ const. In Section 5 we also present lower bounds on $E_{0}$ for all densities. The physical consequences of our findings are resumed in Section 6. Here we provide an argument for the existence of ODLRO in the two and higher dimensional models. It is based on a hypothesis which also permits to estimate the dimension dependence of the deviations of $a_{X}$ from the average $\alpha_{|\partial X|}$ and to conclude that these deviations are irrelevant above two dimensions. In Section 7 we briefly discuss extensions to Hamiltonians with more complicated interactions.

\section{Nonuniformity of the ground state}

The results of this section are valid for any connected, not necessarily regular lattice with all coordination numbers $\leq k$.

Let $A=\left(A_{X Y}\right)$ denote the matrix of $-H_{0}$ in the basis (4). Then $A$ is the adjacency matrix of a graph $G$ whose vertices are the configurations, and two configurations $X$ and $Y$ form an edge of $G$ if and only if their symmetric difference is an edge of $\Lambda: X \backslash Y \cup Y \backslash X=\{x, y\}=\langle x y\rangle$. We denote the largest eigenvalue of $A$ by $\lambda_{1}$ and the corresponding eigenvector by $a=\left(a_{X}\right)$. The ground state energy and wave function are related to them through $E_{0}=-\lambda_{1}$ and $\Psi_{0}=\sum a_{X}|X\rangle$. The coefficients of the ground state wave vector $a$ being positive, $a_{X} / \sum a_{Y}$ can be interpreted as a probability $P_{a}(X)$ of $X$. As we shall see, $P_{a}(X)$ is very far from being a uniform distribution that one can find on complete graphs.

A well-known fact about adjacency matrices is that $\left(A^{n}\right)_{X Y}$ provides the number of walks of length $n$ in $G$ between $X$ and $Y$ (Biggs 1974). Let

$$
W_{n}(X)=\sum_{Y}\left(A^{n}\right)_{X Y}
$$

the number of walks of length $n$ starting from $X$. First, we notice that the expectation value of this number with respect to $P_{a}$,

$$
\left\langle W_{n}\right\rangle_{a} \equiv \sum_{X} W_{n}(X) a_{X} / \sum_{X} a_{X}=\lambda_{1}^{n}=\left\langle W_{1}\right\rangle_{a}^{n}
$$

This follows by taking the $X$ component of the vector equation $A^{n} a=\lambda_{1}^{n} a$, summing over $X$ and dividing by $\sum a_{X}$. The above equality holds true for 
any simple graph: the probability measure $P_{a}$ 'sees' the graph as if it were regular with a degree $\lambda_{1}$.

Next, we make use of the fact that actually we are dealing with a sequence of graphs, $G=G_{\Lambda, N}$, having the particular property that the typical degree is of order $N$ while its change between neighbouring vertices is of order 1: The degree of $X$ is the number of its neighbours, $W_{1}(X)$. There is a oneto-one correspondence between nearest neighbour particle-hole pairs if $X$ is considered as a subset of $\Lambda$ and neighbours of $X$ as a vertex in $G$. Therefore, $\partial X$ can be identified with the set of neighbours of $X$ in $G$ and we have $W_{1}(X)=|\partial X|$. When passing from $X$ to a $Y \in \partial X$ a neighbouring particlehole pair is interchanged. For both the particle and the hole the number of neighbours of the opposite kind can change by at most $k-1$, whence

$$
\left|W_{1}(X)-W_{1}(Y)\right| \leq 2(k-1) \quad \text { if } \quad Y \in \partial X
$$

Now

$$
W_{2}(X)=\sum_{Y \in \partial X}|\partial Y|
$$

which together with (13) yields

$$
\left|W_{1}(X)^{2}-W_{2}(X)\right| \leq 2(k-1)|\partial X| \text {. }
$$

Taking the expectation value and using (12),

$$
0 \leq D_{a}^{2} \equiv\left\langle W_{1}^{2}\right\rangle_{a}-\left\langle W_{1}\right\rangle_{a}^{2} \leq 2(k-1) \lambda_{1} .
$$

By Chebyshev's inequality we then find that for any $\varepsilon>0$

$$
P_{a}\left(\left|W_{1}-\lambda_{1}\right|>\sqrt{2(k-1) \lambda_{1} / \varepsilon}\right)<\varepsilon
$$

or equivalently

$$
\sum_{\left|W_{1}(X)-\lambda_{1}\right| \leq \sqrt{2(k-1) \lambda_{1} / \varepsilon}} a_{X} \geq(1-\varepsilon) \sum a_{X} .
$$

Similarly to (14),

$$
W_{n}(X)=\sum_{Y_{1} \in \partial X} \sum_{Y_{2} \in \partial Y_{1}} \cdots \sum_{Y_{n-1} \in \partial Y_{n-2}}\left|\partial Y_{n-1}\right|
$$


which, together with (13), yields

$$
W_{n}<\prod_{l=0}^{n-1}\left(W_{1}+2(k-1) l\right) \quad W_{n}>\prod_{l=0}^{n-1}\left(W_{1}-2(k-1) l\right) .
$$

These bounds are nontrivial if $n \ll|\Lambda|$, e.g., for $n$ fixed and $|\Lambda|$ going to infinity. (In the opposite limit we have the stronger inequality $W_{n}(X) \leq$ $(k N)^{n}$.) From (20) we get

$$
\left|W_{1}^{n}-W_{n}\right| \leq \sum_{m=1}^{n-1}(2 k-2)^{m}\left(\sum_{1 \leq l_{1}<\cdots<l_{m} \leq n-1} l_{1} \cdots l_{m}\right) W_{1}^{n-m} .
$$

Taking the expectation value,

$$
\left|\left\langle W_{1}^{n}\right\rangle_{a}-\lambda_{1}^{n}\right| \leq \sum_{m=1}^{n-1}(2 k-2)^{m}\left(\sum_{1 \leq l_{1}<\cdots<l_{m} \leq n-1} l_{1} \cdots l_{m}\right)\left\langle W_{1}^{n-m}\right\rangle_{a} .
$$

Replacing $n$ by $n-m$ we obtain

$$
\left\langle W_{1}^{n-m}\right\rangle_{a} \leq \lambda_{1}^{n-m}+O\left(\lambda_{1}^{n-m-1}\right) .
$$

Now $\lambda_{1}$ is of order $|\Lambda|$, so finally

$$
\left|\left\langle W_{1}^{n}\right\rangle_{a}-\lambda_{1}^{n}\right| \leq n(n-1)(k-1)\left[1+O\left(|\Lambda|^{-1}\right)\right] \lambda_{1}^{n-1} .
$$

Equations (16)-(18) and (24) describe the concentration of the probability measure $P_{a}$ on configurations $X$ whose boundary length $|\partial X|$ is in a $\sqrt{N}$ neighbourhood of $\left|E_{0}\right|$. We shall refer to this property as the nonuniformity of the ground state. Summing over $X$ in $\Omega_{n}, P_{a}$ gives rise to the probability distribution $p_{a}$, which is, hence, peaked about $\left|E_{0}\right|$. In Section 4 we shall arrive at the same conclusion in a different way, by studying first the a priori distribution $q(n)=\left|\Omega_{n}\right| / \sum_{m}\left|\Omega_{m}\right|$.

\section{Large deviation principle for the a priori distribution}

The precise computation of $\left|\Omega_{n}\right|$ is a difficult and unsolved combinatorial problem. The logarithm of this number is the entropy of the Ising model, for 
energy $n$, in a microcanonical ensemble with a fixed magnetization, $\sum_{x \in \Lambda} \sigma_{x}=$ $2 N-|\Lambda|$. Indeed, if we put $\sigma_{x}=1$ for $x$ in $X$ and $\sigma_{x}=-1$ elsewhere, we get an Ising configuration with a density $\rho$ of + spins. The corresponding union of contours can be identified with $\partial X$ whose total length $|\partial X|$ is the energy of the Ising configuration. For such systems there exists a strong version of the equivalence of ensembles which, applied to our case, states that the distribution $q$ of $|\partial X|$ satisfies a large deviation principle whose rate function is, apart from a shift, minus the specific entropy $s(e, \rho)$ of the Ising model $(e=n /|\Lambda|)$, cf. Pfister 1991, Dobrushin and Shlosman 1994 and Lewis et al 1994. The exact form of the entropy is unknown. To circumvent this problem, we use an approximate formula for the probability of having a boundary of length $n$,

$$
q(n) \approx Z^{-1} \exp \left\{-\frac{(n-M)^{2}}{2 D^{2}}\right\} .
$$

Here $Z \propto \sqrt{N}$ is for normalization and

$$
M=\overline{|\partial X|}=k \rho(1-\rho)|\Lambda| \quad D^{2}=\overline{(|\partial X|-\overline{|\partial X|})^{2}},
$$

cf. Eq. (10) in the Introduction. The Gaussian approximation is correct in a neighbourhood of the maximum of the specific entropy if the second derivative at the maximum,

$$
\partial^{2} s /\left.\partial e^{2}\right|_{e=k \rho(1-\rho)}=-|\Lambda| / D^{2}
$$

is nonvanishing in the thermodynamic limit. This is what we are going to check by explicitly computing the mean square deviation of $|\partial X|$. We show that

$$
D^{2}=[k-2(2 k-1) \rho(1-\rho)] k \rho(1-\rho)|\Lambda|=[k-2(2 k-1) \rho(1-\rho)] M .
$$

We have found this expression first for $d$-dimensional hypercubic lattices, and checked it also for the triangular, honeycomb and Kagomé lattices. The apparent independence of the details of the lattice is somewhat surprising because our derivation below needs knowledge of the rather different local neighbourhoods up to next-nearest neighbours. The only common feature of all these lattices seems to be that all sites are symmetry-related and thus equivalent. This alone should, therefore, suffice to prove Eq. (28).

Let us remark that, the microcanonical temperature being defined as $(\partial s / \partial e)^{-1}$, the maximum of the entropy corresponds to infinite temperature. 
Therefore, in a neighbourhood of the maximum we are at a safe distance to the ferro- and antiferromagnetic phase transitions, which could invalidate our approximation through a breakdown of the concavity of the microcanonical entropy (Pleimling and Hüller 2000).

Equation (28) is obtained by filling the sites of $\Lambda$ independently and with equal probability $\rho$. We expect smaller order corrections to appear if the computation is done with $N$ fixed, $N /|\Lambda|=\rho$ (cf. Eq. (53) below). So in this section $X$ is a random subset of $\Lambda$ whose probability to be selected is $\rho^{|X|}(1-\rho)^{|\Lambda|-|X|}$, and $n_{x}=n_{x}(X)$ is a random variable taking the value 1 if $x$ is in $X$ and 0 otherwise. Then all $n_{x}$ are independent and take 1 with probability $\rho$ and 0 with probability $1-\rho$.

We define

$$
f_{x}=n_{x} \sum_{y \in \partial x}\left(1-n_{y}\right)
$$

where $\partial x$ denotes the set of neighbours of $x$ in $\Lambda$. The boundary length of $X$ is obtained (cf. Eqs. (5), (6)) as

$$
|\partial X|=\sum_{x \in \Lambda} f_{x}(X) .
$$

Thus the mean value of $(30)$ is

$$
M=\sum_{x \in \Lambda} \overline{f_{x}}=k \rho(1-\rho)|\Lambda|
$$

as claimed earlier.

Let $d(x, y)$ denote the graph distance of $x$ and $y$ in $\Lambda$, i.e., the length of the shortest walk between them. Since $f_{x}$ and $f_{y}$ are independent if $d(x, y)>2$, we find

$$
\begin{gathered}
D^{2}=\sum_{x, y} \overline{\left(f_{x}-\overline{f_{x}}\right)\left(f_{y}-\overline{f_{y}}\right)}=\sum_{x, y: d(x, y) \leq 2} r(x, y) \\
r(x, y)=\overline{f_{x} f_{y}}-{\overline{f_{x}}}^{2} .
\end{gathered}
$$

The computation of the different terms is straightforward by observing that $n_{x}^{2}=n_{x}$. The contribution of the diagonal terms $x=y$ is the same for any $k$-regular lattice. Namely,

$$
\begin{aligned}
\overline{f_{x}^{2}} & =k \rho(1-\rho)+k(k-1) \rho(1-\rho)^{2} \\
\sum_{x \in \Lambda} r(x, x) & =|\Lambda| r(x, x)=\left[k-(2 k-1) \rho+k \rho^{2}\right] M .
\end{aligned}
$$


The contribution of nearest neighbour pairs depends on the number of triangles containing a given edge. If there are $\ell$ such triangles then

$$
\begin{gathered}
\overline{f_{x} f_{y}}=\rho^{2}\left[\ell(1-\rho)+\left[(k-1)^{2}-\ell\right](1-\rho)^{2}\right] \\
\sum_{x, y: d(x, y)=1} r(x, y)=k|\Lambda| r(x, y)=\rho[\ell \rho-(2 k-1)(1-\rho)] M .
\end{gathered}
$$

If $x$ and $y$ are next-nearest neighbours to each other, they may have $m$ common nearest neighbours. Then

$$
\begin{aligned}
\overline{f_{x} f_{y}} & =\rho^{2}\left[m(1-\rho)+\left(k^{2}-m\right)(1-\rho)^{2}\right] \\
r(x, y) & =m \rho^{3}(1-\rho) .
\end{aligned}
$$

In $d$-dimensional hypercubic lattices $(k=2 d)$ there are next-nearest neighbour pairs with $m=1$ and $m=2$. Their contribution to $D^{2}$ is

$$
\begin{aligned}
\sum_{x, y: d(x, y)=2} r(x, y) & =k|\Lambda| r(x, y)_{m=1}+4\left(\begin{array}{l}
d \\
2
\end{array}\right)|\Lambda| r(x, y)_{m=2} \\
& =\rho^{2} M+(k-2) \rho^{2} M=(k-1) \rho^{2} M .
\end{aligned}
$$

For the triangular lattice $(k=6)$

$$
\sum_{x, y: d(x, y)=2} r(x, y)=k|\Lambda|\left[r(x, y)_{m=1}+r(x, y)_{m=2}\right]=(k-3) \rho^{2} M
$$

In the honeycomb lattice $(k=3)$ each site has 6 next-nearest neighbours, all of the type $m=1$. So

$$
\sum_{x, y: d(x, y)=2} r(x, y)=2 k|\Lambda| r(x, y)_{m=1}=(k-1) \rho^{2} M .
$$

In the Kagomé lattice $(k=4)$ there are 8 next-nearest neighbours with $m=1$ :

$$
\sum_{x, y: d(x, y)=2} r(x, y)=2 k|\Lambda| r(x, y)_{m=1}=(k-2) \rho^{2} M .
$$

Finally, we obtain $D^{2}$ by adding (35), (37) with $\ell=0$ and (39) for hypercubic lattices, (35), (37) with $\ell=2$ and (40) for the triangular lattice, (35), (37) with $\ell=0$ and (41) for the honeycomb lattice and (35), (37) with $\ell=1$ and (42) for the Kagomé lattice. All yield (28). 
If the random variables $f_{x}$ were independent, the mean square deviation of their sum would be given by (35). For any $\rho \leq \frac{1}{2}$ this is larger than the actual value (28), so in average the $f_{x}$ 's are negatively correlated. Because $\ell \leq k-1$, the nearest neighbour correlation $r(x, y)<0$ for all $\rho \leq \frac{1}{2}$, cf. Eq. (37). On the other hand, according to (38), for next-nearest neighbours $r(x, y)$ is always positive. Apparently, the induced probability distribution for the $f_{x}$ 's could be approximated by a Boltzmann-Gibbs distribution written with an antiferromagnetic nearest-neighbour Hamiltonian for the centralized variables $f_{x}-\overline{f_{x}}$ and with a temperature and an external field chosen so as to fit the computed average (31) and nearest-neighbour correlations (37). If the effective $\beta$ and field are below their respective critical values we get exponentially decaying antiferromagnetic correlations, and the approximation is qualitatively correct. Adding next-nearest-neighbour interactions one could fit the computed next-nearest-neighbour correlations, and so on.

\section{The form of the averaged wave vector}

Using the large deviation result for $q(n)$ we can obtain more precise informations on $p_{a}(n)$ and $\alpha_{n}$. Apart from normalization $p_{a}(n)$ is obtained by multiplying $q(n)$ with $\alpha_{n}$. Since, as we shall see in the next section, $\lambda_{1}>M$ and the difference is of the order of $N, \alpha_{n}$ has to increase exponentially fast - at least in a neighbourhood of $M$ - so as to shift the expectation value $M$ of $q$ to the expectation value $\lambda_{1}$ of $p_{a}$. As a result, we obtain the approximate expression $p_{a}(n) \sim \exp -\left(n-\lambda_{1}\right)^{2} / 2 D_{a}^{2}$, consistent with our findings in Section 2. This is a second, independent, argument for the nonuniformity of the ground state, assuming the form of another large deviation principle for $|\partial X|$. To get $\alpha_{n}$ we equate this form of $p_{a}(n)$ with the other one, (9), where $\left|\Omega_{n}\right|$ is estimated through (10). This gives

$\alpha_{n} \sim \exp \left(\frac{(n-M)^{2}}{2 D^{2}}-\frac{\left(n-\lambda_{1}\right)^{2}}{2 D_{a}^{2}}\right) \propto \exp \frac{\left(D_{a}^{2}-D^{2}\right) n^{2}+2\left(D^{2} \lambda_{1}-D_{a}^{2} M\right) n}{2 D^{2} D_{a}^{2}}$

For the moment, we have only trivial estimates for $\lambda_{1}(\geq M)$ and $D_{a}$ (cf. Eq. (16)). More precise bounds on $D_{a}$ can be obtained from the monotonic increase of $\alpha_{n}$.

The argument telling that $\alpha_{n}$ should increase for all allowed $n$ is as follows. 
From the spectral decomposition of $A^{m}$ we obtain ( $\operatorname{supposing} \sum a_{X}^{2}=1$ )

$$
a_{X} a_{Y}=\lim _{m \rightarrow \infty} \lambda_{1}^{-m}\left(A^{m}\right)_{X Y}
$$

if $\Lambda$ is non-bipartite, and

$$
a_{X} a_{Y}=\lim _{m \rightarrow \infty} \frac{1}{2}\left[\lambda_{1}^{-m}\left(A^{m}\right)_{X Y}+\lambda_{1}^{-m-1}\left(A^{m+1}\right)_{X Y}\right]=\lim _{m \rightarrow \infty} \frac{\lambda_{1}^{-r_{m}}}{2}\left(A^{r_{m}}\right)_{X Y}
$$

if $\Lambda$ is bipartite; $r_{m}=2 m$ or $2 m+1$ if the graph distance $d_{G}(X, Y)$ of $X$ and $Y$ is even or odd, respectively. Summing over $Y$ and averaging over $X$ in $\Omega_{n}$ we obtain, e.g. for a non-bipartite $\Lambda$,

$$
\alpha_{n}=\left(\sum a_{Y}\right)^{-1} \lim _{m \rightarrow \infty} \lambda_{1}^{-m} \frac{1}{\left|\Omega_{n}\right|} \sum_{X \in \Omega_{n}} W_{m}(X) .
$$

Inspecting Eq. (19) one can conclude that for any $m$ the average of $W_{m}(X)$ over $\Omega_{n}$ increases with $n$ and, hence, $\alpha_{n} \geq \alpha_{n^{\prime}}$ if $n>n^{\prime}$.

Let $n_{\min }$ and $n_{\max }$ denote the smallest and largest allowed values of $|\partial X|$, respectively. Since $n_{\min }=o(N)$, monotonicity of $\alpha_{n}$ implies through (43)

$$
\frac{n_{\max }-\lambda_{1}}{n_{\max }-M} \leq \frac{D_{a}^{2}}{D^{2}} \leq \frac{\lambda_{1}}{M}
$$

up to an error of $o(1)$. Therefore, the coefficient of $n$ in the exponent of $\alpha_{n}$ is nonnegative. Clearly $\lambda_{1} / M=1$ would imply $D_{a} / D=1$ and $\alpha_{n}=$ const. In the next section we show that for finite dimensional lattices $\lambda_{1} / M>1$ asymptotically and, hence, the two bounds in (47) form an interval around 1. On bipartite lattices $n_{\max }=k N$, so at half filling $n_{\max }=2 M$ and 1 is in the centre of the interval. It is possible that $D_{a}=D$ and the exponent of $\alpha_{n}$ is linear in $n$. However, even if $D_{a} \neq D$, the quadratic term in the exponent is of the same order as or even smaller (if $n=o(N)$ ) than the linear term.

More information can be extracted from Eqs. (44) and (45) if we combine them with (18). If $m$ is large enough,

$$
1-2 \varepsilon \leq \frac{1}{W_{m}(X)} \sum_{|| \partial Y\left|-\lambda_{1}\right| \leq \sqrt{2(k-1) \lambda_{1} / \varepsilon}}\left(A^{m}\right)_{X Y} \leq 1
$$

that is, for any $X$ and any sufficiently large $m$ an overwhelming majority of walks of length $m$ starting from $X$ on the graph $G$ end up in a vertex whose degree is $\lambda_{1}+O(\sqrt{N})$. In the expression

$$
W_{m}(X)=\left(a_{X} \sum a_{Y}\right) \lambda_{1}^{m}+o\left(\lambda_{1}^{m}\right) \quad(m \rightarrow \infty),
$$


obtained from (44), $\lambda_{1}^{m}$ accounts for this 'long-time' behaviour. Let us remark that (48) cannot be understood by imagining the walks on $G$ as realizations of some simple random process. Because $G$ is nonregular (unless $\Lambda$ is a complete graph), no Markov process can assign equal probabilities to all walks of equal length. As an example, for a locally unbiased random motion (which chooses among neighbours with equal probability) the most probable individual walks of a given length are the 'descending' ones, those going towards vertices of a lower degree, and the less probable the 'ascending' ones. Because of the form (25) of the distribution of degrees, an $X$ with $|\partial X|<M$ has typically more ascending than descending neighbours and vice versa for $|\partial X|>M$. Therefore, the most probable degrees of end-points of very long walks would be nevertheless close to $M$ (but not to $\lambda_{1}$ ).

Although the monotonicity of $\alpha_{n}$ holds for all $n$ between $n_{\min }$ and $n_{\max }$, the validity of (43) is limited to a neighbourhood of the interval $\left[M, \lambda_{1}\right]$. In particular, the increase of $\alpha_{n}$ for $n=o(N)$ is much steeper than the exponential predicted by (43). Indeed, let us write the eigenvalue equation in the form

$$
\frac{1}{|\partial X|} \sum_{Y \in \partial X} a_{Y}=\frac{\lambda_{1}}{|\partial X|} a_{X}
$$

For a $d$-dimensional lattice $n_{\min }=O\left(N^{\frac{d-1}{d}}\right)$. If we choose $|\partial X|=n$ to be of this order, we find that the average of $a_{Y}$ over $\partial X$ yields $N^{1 / d}$ times $a_{X}$ ! Since $q(n)$ is also rapidly increasing here, the average over $\partial X$ is dominated by $Y$ 's with $n+2 \leq|\partial Y| \leq n+2 k-2$. From this and the monotonicity of $\alpha_{n}$ we conclude that $\alpha_{n+2 k-2} / \alpha_{n}$ is at least of order $N^{1 / d}$.

\section{Bounds on the ground state energy}

\subsection{Variational upper bounds}

Variational estimates of the ground state energy are of the form

$$
E_{0} \leq\langle\psi|H| \psi\rangle /\langle\psi \mid \psi\rangle
$$

A trivial choice is

$$
\psi=\sum|X\rangle
$$


with the summation going over all $N$-point subsets of $\Lambda$. It yields $E_{0} \leq-M$ where

$$
M=\overline{|\partial X|}=\frac{2|E G|}{|V G|}=\frac{2|E \Lambda|\left(\begin{array}{c}
|\Lambda|-2 \\
N-1
\end{array}\right)}{\left(\begin{array}{c}
|\Lambda| \\
N
\end{array}\right)}=k(1-\rho) N+O(1),
$$

cf. Eq. (31). Here $|E G|$ and $|V G|$ denote the number of edges and vertices of $G$, respectively, and $|E \Lambda|$ the number of edges of $\Lambda$. It is not a priori obvious that this bound can be improved in the order of the volume, and it is important to know that it really can. In the opposite case, if $-k \rho(1-\rho)$ were the true ground state energy per site then, as in the complete graph, the Hamiltonian (1) would have a product ground state in infinite volume,

$$
\Psi=\prod_{x}\left(\sqrt{\rho}\left|n_{x}=1\right\rangle+\sqrt{1-\rho}\left|n_{x}=0\right\rangle\right),
$$

i.e., no local perturbation could decrease the energy of $\Psi$. (Because of the product structure, if the energy could be decreased locally, the specific energy $-k \rho(1-\rho)$ of $\Psi$ could also be decreased.) Since $\Psi$ shows ODLRO with the value of the order parameter at its theoretical maximum $(\rho(1-\rho)$, cf. Appendix), by proving it cannot be a ground state we exclude a trivial scenario for Bose-Einstein condensation.

To prove that $\left|E_{0}\right|-M$ is of order $N$ we apply trial functions of the form

$$
\psi_{v}=\sum v_{X}|X\rangle \quad v_{X}=v(|\partial X|)
$$

i.e., $v_{X}$ depending on $|\partial X|$ only. The variational bound (51) reads $\lambda_{1} \geq B(v)$ where

$$
B(v) \equiv \frac{(v, A v)}{(v, v)}=\frac{\sum_{n} v(n) \sum_{i=-k+1}^{k-1} v(n+2 i) \sum_{X \in \Omega_{n}} N_{i}(X)}{\sum_{n} v(n)^{2}\left|\Omega_{n}\right|} .
$$

Here $N_{i}(X)$ is the number of those neighbours of $X$ having a boundary length $|\partial X|+2 i$. In Eq. (56) we have used (13) and the fact that the parity of $|\partial X|$ is the same for all $X$ : even if $N$ is even and that of $k$ if $N$ is odd.

The form (43) of $\alpha_{n}$ suggests that the best choice for $v(n)$ would be $e^{x n+y n^{2}}$ with $x$ of order 1 and $y$ of order $\frac{1}{N}$. However, we do not expect the quadratic term to yield a significant improvement, and choose a simple exponential $v(n)=e^{x n}$ with $0<x<x_{\max }$ where

$$
x_{\max }=\frac{n_{\max }-M}{2 D^{2}} .
$$


Then by making use of the large deviation principle for $|\partial X|$ we find

$$
B(v)=\langle n\rangle_{x}\left\langle e^{2 x i}\right\rangle_{\left[\langle n\rangle_{x}\right]}
$$

where

$$
\langle n\rangle_{x}=\frac{\sum n e^{2 x n}\left|\Omega_{n}\right|}{\sum e^{2 x n}\left|\Omega_{n}\right|} \quad\langle f(i)\rangle_{n}=\sum_{i=-k+1}^{k-1} f(i) \frac{1}{n\left|\Omega_{n}\right|} \sum_{X \in \Omega_{n}} N_{i}(X) .
$$

Now, since $\langle n\rangle_{0}=M$ and $d\langle n\rangle_{x} /\left.d x\right|_{x=0}=2 D^{2}$, we find

$$
\langle n\rangle_{x}=M\left(1+2 x D^{2} / M+O\left(x^{2}\right)\right) .
$$

We notice that the approximate form (10) would yield the same result.

Next, we turn to the second term of $B(v)$. Obviously $\left\langle e^{2 x i}\right\rangle_{n} \geq e^{-2(k-1) x}$. This yields $B(v) / M>1$ for $\rho$ near 0 but not near $\frac{1}{2}$. However, we can use Jensen's inequality $\left\langle e^{2 x i}\right\rangle_{n} \geq e^{2 x\langle i\rangle_{n}}$ together with the fact that $\langle i\rangle_{[M]}$ goes to zero as the volume increases. We then conclude that asymptotically $\langle i\rangle_{\left[\langle n\rangle_{x}\right]}=-$ const $\times x+$ higher order terms, so that the average of the exponential in Eq. (58) equals $1-O\left(x^{2}\right)$. Thus, for $x$ small enough we indeed find $B(v) / M>1$.

With somewhat more effort one can actually compute a lower bound on $B(v)$. We do not make it here, only notice that in optimizing such a bound according to $x$ (and also in optimizing a lower bound on $E_{0}$, see below) the knowledge of $n_{\max }$ is necessary. On bipartite lattices $n_{\max }=k \rho|\Lambda|$ for all $\rho \leq \frac{1}{2}$, and it is an easy graphical exercise to see that the same equality holds for $\rho \leq \frac{1}{3}$ on the triangular and Kagomé lattices. However, for both lattices $n_{\max }$ is constant between the densities $\frac{1}{3}$ and $\frac{1}{2}: 2|\Lambda|$ for the triangular and $\frac{4}{3}|\Lambda|$ for the Kagomé lattice. This can be seen from the following argument. In general, $-n_{\max }$ is the ground state energy of the antiferromagnetic Ising model under the restriction that the magnetization is fixed, $\sum_{x \in \Lambda} \sigma_{x}=(2 \rho-1)|\Lambda|$. However, we do not need to deal with the restriction. In both cases the (unrestricted) ground state is known to be highly degenerate. Among the exponentially large number of ground state configurations there are nonmagnetized ones, corresponding to $\rho=\frac{1}{2}$, others with a concentration of up-spins $\rho=\frac{1}{3}$, and between these two limits $\rho$ can vary by steps of $\frac{1}{|\Lambda|}$. The rule is to flip zero-energy spins one by one. The common energy of all these configurations is easy to compute from the fact that in each triangle there is precisely one unsatisfied bond. This fixes the value of $n_{\max }$ as given above. 
We also remark that the optimal $x$ (which maximizes $B(v)$ ) is small: of order 0.1 or smaller. We shall evoke this fact in the discussion of Section 6 .

\subsection{Lower bounds}

In their paper Dyson, Lieb and Simon (DLS) gave a lower bound on the ground state energy of the spin- $\frac{1}{2}$ XY-model (Dyson et al 1978, Theorem C.1). Because it corresponds to an upper bound on the norm of the Hamiltonian (1) in Fock space, it is automatically valid for the hard-core boson gas (11) at any density. It reads

$$
\left|E_{0}\right| \leq \frac{1}{4}|\Lambda| \begin{cases}\sqrt{k(k+2)} & \text { if } k \text { is even } \\ k+1 & \text { if } k \text { is odd }\end{cases}
$$

The above bound is to be compared with the trivial bound $\left|E_{0}\right| \leq n_{\max }$ obtained by writing Eq. (50) for an $X=X_{0}$ which maximizes $a_{X}$. We then see that (61) is nontrivial for the $d$-dimensional hypercubic lattice if

$\rho>\frac{1}{4} \sqrt{1+\frac{1}{d}}$, for the triangular lattice if $\rho>\frac{1}{2 \sqrt{3}}$, for the honeycomb lattice if $\rho>\frac{1}{3}$ and for the Kagomé lattice if $\rho>\frac{1}{4} \sqrt{\frac{3}{2}}$.

The bound (61) is the best at $\rho=\frac{1}{2}$ and can be improved for lower densities.

If $e_{0}$ is the ground state energy per site then

$$
\left|e_{0}\right|=\left\langle\Psi_{0}\left|A_{x}\right| \Psi_{0}\right\rangle \equiv\left\langle\Psi_{0}\left|\frac{1}{2} \sum_{y \in \partial x}\left(b_{x}^{*} b_{y}+b_{y}^{*} b_{x}\right)\right| \Psi_{0}\right\rangle
$$

for any $x$ in $\Lambda . A_{x}$ preserves the number of bosons in $\Lambda_{x}=\{x\} \cup \partial x$, therefore it commutes with $P_{x, j}$, the orthogonal projection to the subspace $\mathcal{H}_{x, j}$ of states with $j$ particles in $\Lambda_{x}$, where $j=0,1, \ldots, k+1$. We can write

$$
\left\langle\Psi_{0}\left|A_{x}\right| \Psi_{0}\right\rangle=\sum_{j=0}^{k+1} \frac{\left\langle\Psi_{0}\left|P_{x, j} A_{x} P_{x, j}\right| \Psi_{0}\right\rangle}{\left\langle\Psi_{0}\left|P_{x, j}\right| \Psi_{0}\right\rangle}\left\langle\Psi_{0}\left|P_{x, j}\right| \Psi_{0}\right\rangle .
$$

Since $\left\langle\Psi_{0}\left|P_{x, j} \cdot P_{x, j}\right| \Psi_{0}\right\rangle /\left\langle\Psi_{0}\left|P_{x, j}\right| \Psi_{0}\right\rangle$ is a normalized positive linear functional,

$$
\left\langle\Psi_{0}\left|A_{x}\right| \Psi_{0}\right\rangle \leq \sum_{j} \lambda_{\max }(j)\left\langle\Psi_{0}\left|P_{x, j}\right| \Psi_{0}\right\rangle
$$


where $\lambda_{\max }(j)$ is the maximum eigenvalue of $A_{x}$ restricted to $\mathcal{H}_{x, j}$. Now $\lambda_{\max }(j)$ was computed by DLS:

$$
\lambda_{\max }(j)=\frac{1}{2} \sqrt{j(k+1-j)} .
$$

The DLS-bound (61) corresponds to the maximum of 65). Let $h(t)$ be obtained by linear interpolation through the points $\left(j, \lambda_{\max }(j)\right)$ :

$$
h(t)=\frac{1}{2}\{(\lfloor t\rfloor+1-t) \sqrt{\lfloor t\rfloor(k+1-\lfloor t\rfloor)}+(t-\lfloor t\rfloor) \sqrt{(\lfloor t\rfloor+1)(k-\lfloor t\rfloor)}\} .
$$

Since $h(t)$ is a concave function, and the right member of (64) can be written as $\int h(t) d \nu(t)$ with a probability measure $\nu$ concentrated on the integers from 0 to $k+1$, by Jensen's inequality

$$
\left|e_{0}\right| \leq h\left(\int t d \nu(t)\right)=h\left(\sum_{j=0}^{k+1} j\left\langle\Psi_{0}\left|P_{x, j}\right| \Psi_{0}\right\rangle\right) .
$$

The expectation value in the argument of $h$ can be evaluated and yields $(k+1) \rho$. Indeed, using the translation invariance of $\Psi_{0}$, after a simple algebra we find

$$
\sum_{j=0}^{k+1} j\left\langle\Psi_{0}\left|P_{x, j}\right| \Psi_{0}\right\rangle=\sum_{X} a_{X}^{2} \frac{1}{|\Lambda|} \sum_{x \in \Lambda}\left|X \cap \Lambda_{x}\right| .
$$

Let $\chi_{X}$ and $\chi_{\Lambda_{x}}$ denote the characteristic functions of $X$ and $\Lambda_{x}$, respectively. Then

$$
\left|X \cap \Lambda_{x}\right|=\sum_{y \in \Lambda} \chi_{X}(y) \chi_{\Lambda_{x}}(y)
$$

and therefore

$$
\sum_{x \in \Lambda}\left|X \cap \Lambda_{x}\right|=\sum_{y \in \Lambda} \chi_{X}(y) \sum_{x \in \Lambda} \chi_{\Lambda_{x}}(y)=|X| \cdot\left|\Lambda_{x}\right|=N(k+1) .
$$

Hence, we obtain

$$
\left|e_{0}\right| \leq \min \{h((k+1) \rho), k \rho\} \leq \frac{1}{2}(k+1) \sqrt{\rho(1-\rho)}
$$

where the last member results from majorizing $h(t)$ by $\frac{1}{2} \sqrt{t(k+1-t)}$. Taking the minimum in Eq. (71) is not superfluous. For $\rho<\frac{1}{k+1}$ we have 
$h((k+1) \rho)=\frac{1}{2} \sqrt{k}(k+1) \rho$. Since $n_{\max } /|\Lambda|=k \rho$ is smaller for all $k$, the trivial bound is better for small densities. Actually the trivial bound provides the right asymptotics at $\rho=0$ because $e_{0}=-k \rho+O\left(\rho^{2}\right)$ near $\rho=0$, as one can see by comparing the trivial upper and lower bounds, $-M /|\Lambda|$ and $-n_{\max } /|\Lambda|$.

Numerical works on the spin- $\frac{1}{2} \mathrm{XY}$ model in the square lattice yield an approximate formula,

$e_{0}(\rho)=-1.09766+4.835(0.5-\rho)^{2}-1.99232(0.5-\rho)^{4}+0.85952(0.5-\rho)^{6}$.

The first two terms come respectively from a Monte Carlo (Zhang and Runge 1992) and a finite-size scaling (Hamer et al 1999) study, to which we have added the fourth and the sixth order terms to obtain $e_{0}(0)=0$ and $e_{0}^{\prime}(0)=$ -4 . In Figure 1 we have plotted the trivial upper bound $-4 \rho(1-\rho)$, the formula (72) and the lower bound $-\min \{h((k+1) \rho), k \rho\}$. We have also shown numerical points for a $5 \times 5$ lattice from Table 1 of Hamer et al 1999 . They nicely follow the curve (72).

Using the formula (72) we can compute the lower and upper bounds (47) on $D_{a}^{2} / D^{2}$. Both of them increase monotonically as $\rho$ varies between 0 and 0.5 : the lower bound from $\sim 0.66$ to 0.90234 and the upper bound from 1 to 1.09766, their difference decreasing monotonically at the same time.

\section{Off-diagonal order and the final form of the ground state}

Off-diagonal long-range order in the ground state is characterized by (cf. Appendix)

$$
\omega_{0} \equiv \lim \frac{1}{|\Lambda|^{2}} \sum_{X} a_{X} \sum_{x \in X} \sum_{y \notin X} a_{X \cup\{y\} \backslash\{x\}}>0
$$

where lim means the thermodynamic limit $(|\Lambda|$ and $N$ going to infinity and $N /|\Lambda|$ going to $\rho$ ). In (73) $\sum a_{X}^{2}=1$ is supposed.

Let us see, what would we get if $a_{X}$ would not fluctuate within $\Omega_{n}$, i.e., if $a_{X}=\alpha_{|\partial X|}$. This and (43) would imply another large deviation principle in the approximate form

$$
\sum_{X \in \Omega_{n}} a_{X}^{2}=\alpha_{n}^{2}\left|\Omega_{n}\right| \sim \exp \left[-\frac{\left(n-\lambda_{2}\right)^{2}}{2 D_{a^{2}}^{2}}\right]
$$




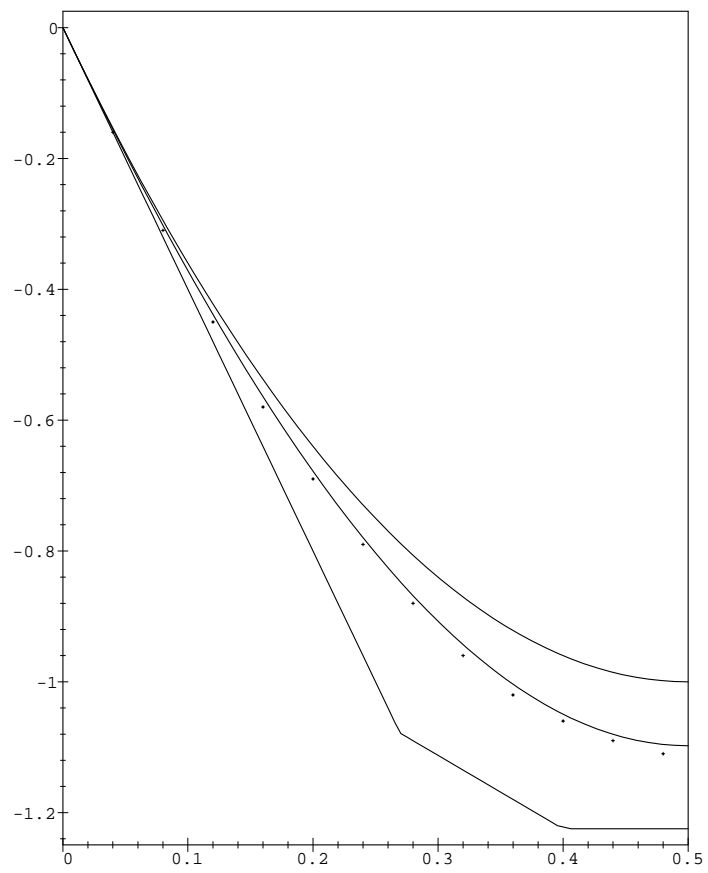

Figure 1: Approximations of the ground state energy per site as a function of the density in the square lattice. 
with

$$
\lambda_{2}=\lambda_{1}+D_{a}^{2} \frac{\lambda_{1}-M}{2 D^{2}-D_{a}^{2}} \quad D_{a^{2}}^{2}=\frac{D^{2} D_{a}^{2}}{2 D^{2}-D_{a}^{2}} .
$$

The mean value $\lambda_{2}$ is larger than $\lambda_{1}$ because

$$
\frac{D_{a}^{2}}{D^{2}} \leq \frac{\lambda_{1}}{M}<\min \left\{\frac{1}{1-\rho}, \frac{k+1}{2 k \sqrt{\rho(1-\rho)}}\right\}<2
$$

see Eq. (71). As

$$
\|\partial(X \cup\{y\} \backslash\{x\})|-| \partial X\| \leq 2 k,
$$

with $a_{X \cup\{y\} \backslash\{x\}} \geq \alpha_{|\partial X|-2 k}$ we would find

$$
\omega_{0} \geq \lim e^{-\frac{2 k\left(\lambda_{1}-M\right)}{2 D^{2}-D_{a}^{2}}}>0 .
$$

The argument above, together with the conclusion (78) may be right in high enough dimensions but it is known to be incorrect in one dimension. Fluctuations have to be taken into account.

A way to prove (73) would be to show

$$
a_{X \cup\{y\} \backslash\{x\}} \geq c a_{X}
$$

for all $X, x$ and $y$ with a $c>0$ independent of $\Lambda$ and $N$. If (79) would hold true, we would find $\omega_{0} \geq c \rho(1-\rho)>0$. In the complete graph (79) is verified with $c=1$ and equality sign. However, we know already from the considerations following Eq. (50) that in finite dimensional lattices (79) cannot hold indeed for all $X$ : e.g., in the case when $|\partial X| \propto N^{1-\frac{1}{d}}$, (79) can be satisfied only with $c \propto N^{-\frac{1}{d}}$. Also, we should not be able to prove (79) in one dimension. Below we present an argument which takes into account fluctuations and distinguishes between one, two and higher dimensions.

First, we notice that we need (79) only for $X$ in a subset $S(\Lambda, N)$ such that the sum of $a_{X}^{2}$ over $S$ is nonvanishing in the thermodynamic limit. Let us introduce a function $R(X)$ by setting $a_{X}=\alpha_{|\partial X|} e^{R(X)}$. Then

$$
\sum_{X \in \Omega_{n}} e^{R(X)}=\left|\Omega_{n}\right| \leq \sum_{X \in \Omega_{n}} e^{2 R(X)} \leq\left|\Omega_{n}\right|^{2},
$$

so we can define an $\epsilon_{n}$ such that $0 \leq \epsilon_{n} \leq 1$ and

$$
\sum_{X \in \Omega_{n}} e^{2 R(X)}=\left|\Omega_{n}\right|^{1+\epsilon_{n}} .
$$


Using this and Eq. (43) we find

$$
\sum_{X \in \Omega_{n}} a_{X}^{2}=\alpha_{n}^{2}\left|\Omega_{n}\right|^{1+\epsilon_{n}} \sim \exp \left[-\frac{\left(n-M_{n}\right)^{2}}{2 D_{n}^{2}}\right]
$$

with

$$
M_{n}=\lambda_{1}+\frac{\left(1-\epsilon_{n}\right) D_{a}^{2}}{2 D^{2}-\left(1-\epsilon_{n}\right) D_{a}^{2}}\left(\lambda_{1}-M\right) \quad D_{n}^{2}=\frac{D^{2} D_{a}^{2}}{2 D^{2}-\left(1-\epsilon_{n}\right) D_{a}^{2}} .
$$

Because of the possible $n$-dependence of $M_{n}$ and $D_{n}$, Eq. (82) may not describe a large deviation principle. However, $M_{n}$ and $D_{n}$ satisfy the inequalities $\lambda_{1} \leq M_{n} \leq \lambda_{2}$ and $D_{a} / \sqrt{2} \leq D_{n} \leq D_{a^{2}}$ and, hence, for any $\varepsilon>0$

$$
\lim \sum_{(1-\varepsilon) \lambda_{1} \leq|\partial X| \leq(1+\varepsilon) \lambda_{2}} a_{X}^{2}=1
$$

For $S$ we can take the set of summation with any $\varepsilon \geq 0$.

Next, we observe that by using Eqs. (44) and (45) we can write

$$
\frac{a_{X}}{a_{Y}}=\lim _{m \rightarrow \infty} \frac{W_{m}(X)}{W_{m}(Y)} \quad \text { or } \quad \lim _{m \rightarrow \infty} \frac{\lambda_{1} W_{m}(X)+W_{m+1}(X)}{\lambda_{1} W_{m}(Y)+W_{m+1}(Y)}
$$

for non-bipartite or bipartite lattices, respectively. The convergence in (85) is usually slow. However, we expect that if $|\partial X|$ and $|\partial Y|$ do not differ too much then, at least up to the order of magnitude, $a_{X} / a_{Y}$ can be approximated by the ratio of the number of walks whose length is the distance between $X$ and $Y$. Thus, we conjecture that for $|\partial X|=c_{1} N$ and $\|\partial Y|-| \partial X\| \leq c_{2}$

$$
\left|\ln \frac{a_{X}}{a_{Y}}-\ln \frac{W_{d_{G}(X, Y)}(X)}{W_{d_{G}(X, Y)}(Y)}\right| \leq c_{3}
$$

where $c_{3}$ may depend on the constants $c_{1}$ and $c_{2}$ but not on the size of the system. In what follows, we examine the consequences of this hypothesis.

If the aspect ratios of $\Lambda$ are kept bounded, its diameter $L$ is of order $|\Lambda|^{\frac{1}{d}}$ and the diameter of $G$ is of order $L^{d+1}$. Over such a distance (86) allows a gigantic change of $a_{X}$. However, the distance to be considered for (79) is much smaller. If the symmetric difference of $X$ and $Y$ is $\{x, y\}$, it is easily seen that $d_{G}(X, Y)=d(x, y) \leq L$. Let $|\partial X|=n \propto N$. Above one dimension we apply the inequalities (20) to obtain

$$
\frac{W_{d_{G}(X, Y)}(X)}{W_{d_{G}(X, Y)}(Y)} \leq\left(\frac{1+\frac{2(k-1) L}{n}}{1-\frac{2(k-1) L+2}{n}}\right)^{L} .
$$


In the thermodynamic limit the upper bound remains finite in two dimensions and converges to 1 in higher dimensions, so we get the necessary estimate (79). In one dimension there exists no convergent upper bound. Thus, we find ODLRO in the ground state in two and higher dimensions but not in one dimension.

The difference between 2 and $2+\varepsilon$ dimensions in the convergence of the right-hand side of Eq. (87) indicates that $d=2$ is the critical dimension above which the deviations of $a_{X}$ from the average $\alpha_{|\partial X|}$ are irrelevant. This remark can be made quantitative if we observe an interesting consequence of the hypothesis (86) on $R(X)$. If $|\partial X| \propto N$ then inside a sphere of radius $\propto \sqrt{N}$, centred at $X$, for all $Y$ such that ||$\partial Y|-| \partial X||$ is bounded by some constant of order of unity $|R(Y)-R(X)|$ also remains below another constant of order 1. Comparing the scale $\sim \sqrt{N}$ with the diameter $\sim N^{1+1 / d}$ of $G$ we conclude that

$$
|R(X)| \leq \max R(Y)-\min R(Y)<\text { const } \times N^{\frac{1}{2}+\frac{1}{d}} .
$$

The maximum and the minimum are taken over $\Omega_{|\partial X|}$. The first inequality holds because for any $n$ the average of $R(X)$ over $\Omega_{n}$ is negative, but $R(X)$ cannot be negative for all $X$.

According to Equation (88),

$$
a_{X}=\exp [F(|\partial X|)+R(X)]=\exp \left[F(|\partial X|)+O\left(N^{\frac{1}{2}+\frac{1}{d}}\right)\right] .
$$

Therefore, in one dimension $R(X)$ can dominate $F(|\partial X|)$, in two dimensions it can vary on the same scale $\sim N$, and above two dimensions the variation of $R(X)$ is negligible as compared to that of $F(|\partial X|)$. Apart from an additive constant necessary for normalization, the approximate form of $F(n)=\ln \alpha_{n}$ can be read off Eq. (43).

The upper bound in the right member of Eq. (88) could, of course, overestimate the order of magnitude of the maximum of $|R(X)|$. However, because it seems to correctly distinguish between one, two and higher dimensions, it is probably sharp. This implies also that on the scale $\sim \sqrt{N}$ the function $R(X)$ can be considered as describing rather a ballistic motion over $G$ than a random fluctuation about a negative average, which would lead to a smaller bound. Accepting ballisticity on smaller scales as well, one can find the order of magnitude of local variations of $R(X)$. We distinguish between two cases:

(i) If $\Lambda$ is bipartite with sublattices $V_{1}, V_{2}$ then $G$ is also bipartite with sublattices $\Gamma_{1}=\left\{X:\left|X \cap V_{1}\right|\right.$ is even $\}$ and $\Gamma_{2}=\left\{X:\left|X \cap V_{1}\right|\right.$ is odd $\}$. 
Along each path in $G$ the subsequent terms belong to alternating sublattices. If $\partial^{2} X$ denotes the set of second neighbours of $X$ then

$$
|R(X)-R(Y)|<\text { const } \times N^{-1 / 2} \quad \text { if } Y \in \partial^{2} X \text { and }|\partial Y|=|\partial X| .
$$

(ii) If $\Lambda$ is not bipartite, neither is $G$, and no systematic compensation of terms of different signs is possible. In this case

$$
|R(X)-R(Y)|<\text { const } \times N^{-1 / 2} \quad \text { if } Y \in \partial X \text { and }|\partial Y|=|\partial X| \text {. }
$$

The result on ODLRO could be obtained from (90), (91) as well.

The two-dimensional case being marginal, some subtle logarithmic corrections, that we do not see, may modify our conclusions.

Above two dimensions we also find, as a further consequence of Eq. (88), that $\epsilon_{n}$ identically vanishes in the thermodynamic limit. Thus, $M_{n} / \lambda_{2} \rightarrow 1$ and $D_{n} / D_{a^{2}} \rightarrow 1$, Eq. (82) asymptotically coincides with Eq. (74), Eq. (78) provides a valid lower bound on the order parameter, and Eq. (84) can be replaced by

$$
\lim \sum_{(1-\varepsilon) \lambda_{2} \leq|\partial X| \leq(1+\varepsilon) \lambda_{2}} a_{X}^{2}=1
$$

for any $\varepsilon>0$. This property makes $a_{X}^{2}$ similar to a thermal Gibbs state for a classical lattice gas. The classical Hamiltonian (with $1 / k_{B} T$ incorporated) is $H_{\text {eff }}(X)=-2 F(|\partial X|)-2 R(X)$, and there is asymptotic equivalence between the canonical distribution and a microcanonical one concentrated on $X$ 's with $H_{\text {eff }}(X)$ in an $o(N)$ neighbourhood of the canonical expectation value of $H_{\text {eff }}$. This holds in spite of $H_{\text {eff }}$ containing long-range interactions. Indeed, $-F$ is a sum of nearest neighbour repulsive two-body interactions, $f_{x}$, and, if $D_{a} \neq D$, properly normalized long-range four-body interactions, $f_{x} f_{y}$, see Eqs. (29), (30) and (43), the whole yielding a global repulsion which tends to maximize $|\partial X|$. Now $R$ may also include up to $N$ body interactions but because $R(X)=o(N)$ and because $a_{X}^{2}$ corresponds to a high-temperature Gibbs state, it can be neglected: The fact that $R$ may partly split the ground state degeneracy of $-F$ is irrelevant at high temperatures.

We have no rigorous proof that the limiting effective Gibbs state is a pure (in particular, a high-temperature) state. For this we should show that for all densities and all relevant $n, 2 F(n) / n<K_{c}$, the critical coupling of the Ising model on the same lattice. Even if we knew $\lambda_{1}$ exactly, our bounds (47) on $D_{a} / D$ are not good enough to obtain this information. For example, in the case of the square lattice we can use the numerical fit (72). If we assume that 
$D_{a} \geq D$, we get $2 F(n) / n \leq 2\left(\lambda_{1}-M\right) / D^{2}$ where the upper bound increases with $\rho$ and its maximum at $\rho=0.5$ is $0.3906<K_{c}=0.4407$. On the other hand, if we use the lower bound for $D_{a}$, at half filling we find for $n \geq \lambda_{1}$ that $2 F(n) / n \leq 0.628>K_{c}$. An indication to the high-temperature character can be found in the variational estimates, cf. the final remark of Section 5.1. In the light of Eq. (89), trial functions of the form (55) acquire a particular importance: For $d>2$ one could find with them the exact ground state energy per site, and the optimal exponential ansatz may not be far from the true ground state. The variable $x$ appearing in the formula (58) corresponds to $\frac{1}{2} \beta J$ of the Ising model. The estimates we have done predict a value well below the critical one in any dimension: $\beta J<0.2$ for all densities at $k=3$, and decreases with increasing $k$, reaching 0 in the complete graph.

Now $a_{X}^{2}$ being equivalent to a high-temperature Gibbs state means that there is no classical (diagonal) order in the ground state, coexisting with the purely quantum-mechanical (off-diagonal) one. Diagonal order is characterized by an order parameter operator $O$ which is diagonal in the basis (4) and for which the ground state expectation value $|\Lambda|^{-2}\left\langle\Psi_{0}\left|O^{*} O\right| \Psi_{0}\right\rangle=$ $|\Lambda|^{-2} \sum a_{X}^{2}|O(X)|^{2}$ has a nonvanishing thermodynamic limit. A typical example is $O_{q}=\sum_{x} e^{i(q, x)} n_{x}$, associated to a periodic order with a wave vector $q$. Due to the correspondence with a Gibbs state, diagonal order in the ground state of the Bose gas is equivalent to low-temperature order in the associated classical lattice gas. The ground state of $-F$ is exponentially degenerate for $\rho<1 / 2$. However, it is only two-fold degenerate on bipartite lattices at $\rho=1 / 2$, and the degeneracy is not split by the translation invariant $R(X)$. Apparently, there is no qualitative argument against a crystalline order at half filling in the ground state of the $d>2$ dimensional purely hard-core Bose gas. The nearest neighbour repulsion in $-F$ is simply not strong enough, reflecting the fact that a purely on-site repulsion in $H_{0}$, even though infinite, cannot induce such an order.

\section{Extensions and concluding remarks}

The nonuniformity of the ground state we have found in a purely hardcore gas seems to be a general property of finite-range Hamiltonians. The extension to more complicated hard-core Hamiltonians is immediate. Let

$$
H=-\sum_{\langle x y\rangle}\left(b_{x}^{*} b_{y}+b_{y}^{*} b_{x}\right)+\sum V_{x} n_{x}-J \sum_{\langle x y\rangle}\left(n_{x}-\frac{1}{2}\right)\left(n_{y}-\frac{1}{2}\right)+K\left(\left\{n_{x}\right\}\right)
$$




$$
\equiv H_{0}+V+\frac{J}{4}(2 \Delta-|E \Lambda|)+K
$$

where $\Delta$ is the diagonal part of $H_{0}^{2}$, cf. Eq. (6), and $K$ may contain further interactions of a bounded range. Because the terms additional to $H_{0}$ are diagonal in the basis (田), the Perron-Frobenius theorem still applies and the ground state wave vector $a_{X}>0$. If $A$ is the matrix of $-H$ then keeping the definition (11) we still have (12), and the inequalities of Section 2 also hold with suitable modifications. In particular, in (13) and (15) $2(k-1)$ is to be replaced by $\kappa=|2-J|(k-1)+\delta V+\delta K$ where $\delta V=\max _{\langle x y\rangle}\left|V_{x}-V_{y}\right|$ and $\delta K=\max _{X, Y \in \partial X}|K(Y)-K(X)|$ are of the order of unity, and in (16)-(18) we have $\kappa\langle|\partial X|\rangle_{a}$ instead of $2(k-1) \lambda_{1}$. Now

$$
W_{1}(X)=A_{X X}+|\partial X|=\left(1-\frac{J}{2}\right)|\partial X|-V(X)-K(X)+\frac{J}{4}|E \Lambda|
$$

may not be positive and $\left\langle W_{1}\right\rangle_{a}=\lambda_{1}=-E_{0}$ may not be of order $N$. Therefore, there may not be a large deviation principle for the pair $\left(W_{1}, a\right)$. However, if we replace $H$ by $H^{c}=H+c I$ where $I$ is the identity operator, the ground state vector $a$ will not change. The corresponding $W_{1}$ is $W_{1}^{c}(X)=W_{1}(X)-c$, and $D_{a}^{2}\left(W_{1}^{c}\right)$ is also independent of $c$. Let us choose $c=\left\langle A_{X X}\right\rangle_{a}$, then

$$
\lambda_{1}+c \equiv \lambda_{1}^{c}=\left\langle W_{1}^{c}\right\rangle_{a}=\langle|\partial X|\rangle_{a}
$$

and, thus, we have a large deviation principle for $\left(W_{1}^{c}, a\right)$ in the same form as for the purely hard-core interaction.

What really counts for the nonuniformity of $a$ is not the large deviation principle for $\left(W_{1}, a\right)$ but the variation of $W_{1}(X)$ over the set of $N$-point configurations. The ground state is nonuniform if $\left[\max W_{1}(X)-\min W_{1}(X)\right] / \sqrt{N}$ goes to infinity with $N$. As an example, let us consider the nearest neighbour anisotropic Heisenberg model $(V=K=0)$. At $J=2$, when it is isotropic, for any fixed $N$ we have $W_{1}(X) \equiv|E \Lambda| / 2$ and hence $\lambda_{1}=|E \Lambda| / 2$ and $a_{X}$ is constant. Away from the isotropy point $\Delta W_{1}$ is of order $N$ and $a$ is nonuniform.

If $\Omega_{n}=\left\{X: W_{1}(X)=w_{n}\right\}$, where $w_{1}<w_{2}<\ldots$ are the possible values of $W_{1}$, one can write down an approximate formula similar to Eq. (10), namely $q(n) \sim \exp \left[-\left(w_{n}-\overline{W_{1}}\right)^{2} / 2 D^{2}\left(W_{1}\right)\right]\left(w_{n+1}-w_{n}\right)$. Interpreting $\alpha_{n}$ as the average of $a_{X}$ over the newly defined $\Omega_{n}$, we have also the analogue of Eq. (43). 
As earlier, we can write $a_{X}=\exp \left[F\left(W_{1}(X)\right)+R(X)\right]$, and Eq. 80 remains valid. The approximate form of $F$ is

$$
F\left(W_{1}(X)\right) \approx \frac{\left(W_{1}(X)-\overline{W_{1}}\right)^{2}}{2 D\left(W_{1}\right)^{2}}-\frac{\left(W_{1}(X)+E_{0}\right)^{2}}{2 D_{a}\left(W_{1}\right)^{2}}+\text { const } .
$$

One can replace the infinite on-site repulsion by a finite one provided that stability is assured, $H>-$ const $\times N$. The dimension of the $N$-particle subspace will increase to $\left(\begin{array}{c}|\Lambda|+N-1 \\ N\end{array}\right)$. A configuration $X$ becomes a list of $N$ elements containing vertices of $\Lambda$ with possible repetitions that we can write as $X=\left\{x^{n_{x}}\right\}_{x \in \Lambda}$. The neighbourhood relation remains the same: $Y \in \partial X$ if $Y$ can be obtained from $X$ by moving a single particle along an edge of $\Lambda$, say, from $x$ into $y$. The corresponding matrix element of $-H$ is then $A_{X Y}=\sqrt{n_{x}(X) n_{y}(Y)}$. Now $\sum_{Y \neq X} A_{X Y}$ can vary between $k \sqrt{N}$ and $\sqrt{2} k N$ when $|\partial X|$ varies between $k$ and $k N$. However, $A_{X X}$ can be of order $N^{2}$ and its change between neighbours of order $N$. Therefore, we miss a pointwise bound like (13) and cannot repeat the argument of Section 2. Nevertheless, $-W_{1}(X)=\sum_{Y}\langle Y|H| X\rangle$ is still the energy of a classical lattice gas in which the equivalence of the canonical and microcanonical ensembles implies the analogue of Eq. (10) and, via the argument of Section 4, the nonuniformity of the ground state.

The real novelty introduced by the additional interactions is the possible coexistence of classical long-range order with the off-diagonal one. A thorough discussion of this question, concluding negatively, can be found already in Penrose and Onsager's 1956 paper. Nevertheless, a controversy has remained until recently, when reliable quantum Monte Carlo computations on square lattice models have shown such a coexistence (Batrouni et al 1995). We hope that the ideas developed in this paper can contribute in the future to further elucidate this interesting problem.

This work was supported by the Hungarian Scientific Research Fund (OTKA) under Grant No. T 30543.

\section{Appendix. A digression on ODLRO}

Following the standard definition (Penrose and Onsager 1956, PO), we give below the expression of the ODLRO parameter in terms of $\left(a_{X}\right)$, valid for any Bose gas whose Hamiltonian contains a hard-core repulsion and conserves the 
number of particles. The density operator in the ground state $\Psi_{0}$ (supposed to be normalized) is the orthogonal projection $\left|\Psi_{0}\right\rangle\left\langle\Psi_{0}\right|$. The density matrix in the basis (4) is therefore $\left(a_{X} a_{Y}\right)$. From here the one-particle reduced density matrix $\sigma=\left(\sigma_{x y}\right)$ is obtained by taking a partial trace over $N-1$ particle positions,

$$
\sigma_{x y}=\sum_{X^{\prime}} a_{X^{\prime} \cup\{x\}} a_{X^{\prime} \cup\{y\}} .
$$

In the above sum $X^{\prime}$ runs over the $N$-1-point subsets of $\Lambda$ not containing $x$ and $y$. The matrix $\sigma$ is real symmetric, positive semidefinite (in fact, positive definite for $N>2$ ), its trace is $N$ and all its elements are positive. A possible choice for the order parameter, according to $\mathrm{PO}$, is

$$
\omega=|\Lambda|^{-2} \sum_{x, y \in \Lambda} \sigma_{x y}
$$

Another definition of PO for the order parameter is $1 /|\Lambda|$ times the largest eigenvalue of $\sigma$. If $\Lambda$ is a shift-invariant set (which supposes periodic boundary conditions), the ground state is translation invariant and $\sigma_{x y}=\sigma_{x-y}$. Applying the Perron-Frobenius theorem to $\sigma$ we find that its largest eigenvalue is $\sum_{y} \sigma_{x-y}=|\Lambda| \omega$, so that the above two definitions coincide. The order parameter $\omega$ has also an interpretation as the density of the Bose-Einstein condensate. This holds because the operator associated to the density of the condensate is

$$
|\Lambda|^{-1} b_{k=0}^{*} b_{k=0}=|\Lambda|^{-2} \sum_{x, y \in \Lambda} b_{x}^{*} b_{y}
$$

and because

$$
\left\langle\Psi_{0}\left|b_{x}^{*} b_{y}\right| \Psi_{0}\right\rangle=\sigma_{x y} .
$$

Inserting (97) into (98) we obtain

$$
\omega=\omega^{\prime}+\frac{N}{|\Lambda|^{2}} \quad \omega^{\prime}=\frac{1}{|\Lambda|^{2}} \sum_{X} a_{X} \sum_{x \in X} \sum_{y \notin X} a_{X \cup\{y\} \backslash\{x\}} .
$$

The maximum of $\omega^{\prime}$ under the condition of normalization is $\rho(1-\rho)$, attained with $a_{X}$ constant. It is realized by $H_{0}$ on complete graphs and by the ferromagnetic Heisenberg model on arbitrary graphs. Our $\omega^{\prime}$ is one fourth of the order parameter usually used in the spin- $\frac{1}{2}$ XY model, cf. Fujiki and Betts 1986, Hamer et al 1999. There is ODLRO for a density $\rho$ if $\omega$ or, equivalently, $\omega^{\prime}$ has a nonvanishing limit $\omega_{0}>0$ as $N$ and $|\Lambda|$ go to infinity 
while $N /|\Lambda|$ goes to $\rho$. When this occurs, conservation of the particle number is spontaneously broken and there appears (at least) a one-parameter continuum of ground states in infinite volume. The order parameter operator, $\sum b_{x}^{*}$, is nondiagonal in the natural basis (四) (whence the name ODLRO). The ground states $\gamma_{\alpha}, 0 \leq \alpha<2 \pi$, are symmetry-related. For any local operator $B$

$$
\gamma_{\alpha}(B)=\gamma_{0}\left(\prod_{x} e^{-i \alpha n_{x}} B \prod_{x} e^{i \alpha n_{x}}\right)
$$

In particular,

$$
\gamma_{\alpha}\left(b_{x}^{*}\right)=e^{-i \alpha} \gamma_{0}\left(b_{x}^{*}\right) \neq 0
$$




\section{References}

[1] Batrouni G G, Scalettar R T, Zimanyi G T and Kampf A P 1995 Phys. Rev. Letters $\mathbf{7 4} 2527$

Scalettar R T, Batrouni G G, Kampf A P and Zimanyi G T 1995

Phys. Rev. B 518467

[2] Biggs N 1974 Algebraic Graph Theory (Cambridge University Press)

[3] Dobrushin R L and Shlosman S B 1994 Adv. Sov. Math. 2091

[4] Dyson F J, Lieb E H and Simon B 1978 J. Stat. Phys. 18335

[5] Fujiki S and Betts D D 1986 Can J. Phys. 64876

[6] Hamer C J, Hövelborn T and Bachhuber M 1999

J. Phys. A: Math. Gen. 3251

[7] Kennedy T, Lieb E H and Shastry B S 1988 Phys. Rev. Letters 612583

[8] Kubo K and Kishi T 1988 Phys. Rev. Lett. 612585

[9] Lewis J T, Pfister C E and Sullivan W G 1994 J.Stat. Phys. 77397

[10] Matsubara T and Matsuda H 1956 Prog. Theor. Phys. 16569

[11] Penrose O 1991 J. Stat. Phys. 63761

[12] Penrose O and Onsager L 1956 Phys. Rev. 104576

[13] Pfister C E 1991 Helv. Phys. Acta 64953

[14] Pleimling M and Hüller A 2000 arXiv:cond-mat/0011116

[15] Tóth B 1991 J. Stat. Phys. 65373

[16] Tóth B 1990 J. Stat. Phys. 61749

[17] Yang C N 1962 Rev. Mod. Phys. 34694

[18] Zhang S and Runge K 1992 Phys. Rev. B 451052 